\title{
Congenital Arteriovenous Malformation of Lip - A Case Report
}

\author{
Rajasbala Pradeep Dhande ${ }^{1}$, Megha Manoj², Roohi Gupta 3 , Prerna Patwa ${ }^{4}$, Prasanthi Ghanta ${ }^{5}$ \\ 1, 2,3,4,5 Department of Radiodiagnosis, Jawaharlal Nehru Medical College, Datta \\ Meghe Institute of Medical Sciences (DMIMS), Wardha, Maharashtra, India.
}

\section{INTRODUCTION}

Vascular anomalies are a heterogeneous group of lesions involving vascular channels including the lymphatics. They encompass a wide variety of lesions from simple capillary haemangiomas to angiosarcomas. These lesions most commonly occur as a result of developmental error during embryogenesis due to defective signal process. ${ }^{1}$ Most of these lesions occur sporadically while a few may be inherited or acquired. Inherited lesions tend to be small and multi-centric which gradually increase its size with age. ${ }^{2}$ The International Society for the Study of Vascular Anomalies has broadly classified vascular anomalies into 2 groups: 1) Vascular neoplasms and 2) Vascular malformations. ${ }^{3}$ Vascular malformations are a relatively rare group of lesions involving the endothelium and surrounding tissue of arteries and veins resulting in an abnormal arteriovenous shunting. They are categorised into 4 types: 1) Venous malformation, 2) Capillary malformation, 3) Arteriovenous malformation and 4) Lymphatic malformation. They can occur anywhere in the body from head to toe, but they are most commonly seen in the brain. The most common extra-cranial site for $\mathrm{AV}$ malformations is the head and neck and other common sites include limbs, trunk and viscera. ${ }^{4}$ Here, we a present a rare case of congenital AV malformation of lip in a 49-year-old male.

\section{PRESENTATION OF CASE}

A 49-year-old male presented to the Department of Surgery with complaint of a gradually increasing swelling in the upper lip since 45 years. Past history revealed an episode of bleeding from the lesion. There was no history of fever or trauma. On local examination, the swelling in the upper lip was found to be soft, fluctuant and painless (Figure 1). The swelling was pulsatile in nature. A similar swelling was also found on the dorsum of the nose.
Corresponding Author: Dr. Megha Manoj, Jawaharlal Nehru Medical College, Datta Meghe Institute of Medical Sciences (DMIMS), Wardha, Maharashtra, India. E-mail:meghamanoj17@gmail.com

DOI: $10.14260 / \mathrm{jemds} / 2021 / 482$

How to Cite This Article:

Dhande RP, Manoj M, Patil LB, et al. Congenital Arteriovenous malformation of lip - a case report. J Evolution Med Dent Sci 2021;10(30):2354-2357, 10.14260/jemds/2021/482

Submission 07-05-2021, Peer Review 05-07-2021, Acceptance 12-07-2021, Published 26-07-2021.

Copyright (c) 2021 Rajasbala Pradeep Dhande et al. This is an open access article distributed under Creative Commons Attribution License [Attribution 4.0 International (CC BY 4.0)] 


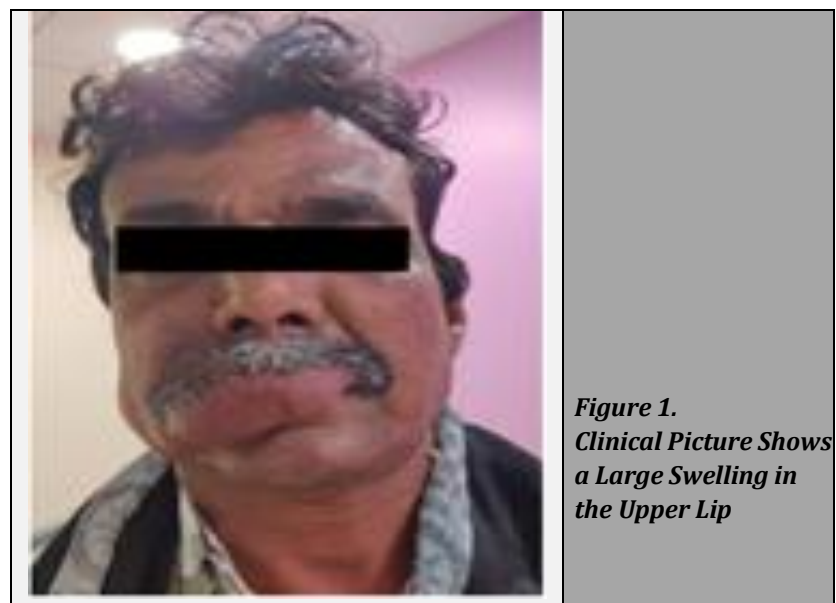

A provisional diagnosis of a vascular lesion was made and the patient was referred to the Department of Radio-diagnosis for ultrasound examination and contrast-enhanced CT scan of head and neck. An ultrasound examination of the lesion was done (Figure 2, 3). On ultrasound, there was evidence of a heterogeneous lesion with multiple tubular anechoic structures within in the upper lip. On Colour Doppler Imaging (CDI), the tubular anechoic structures showed colour flow with mixing of red and blue colours. Spectral waveform showed a high velocity flow with low resistance within the nidus. The ultrasound findings of the lesion were found to be consistent with the clinical diagnosis of a vascular lesion - AV malformation.
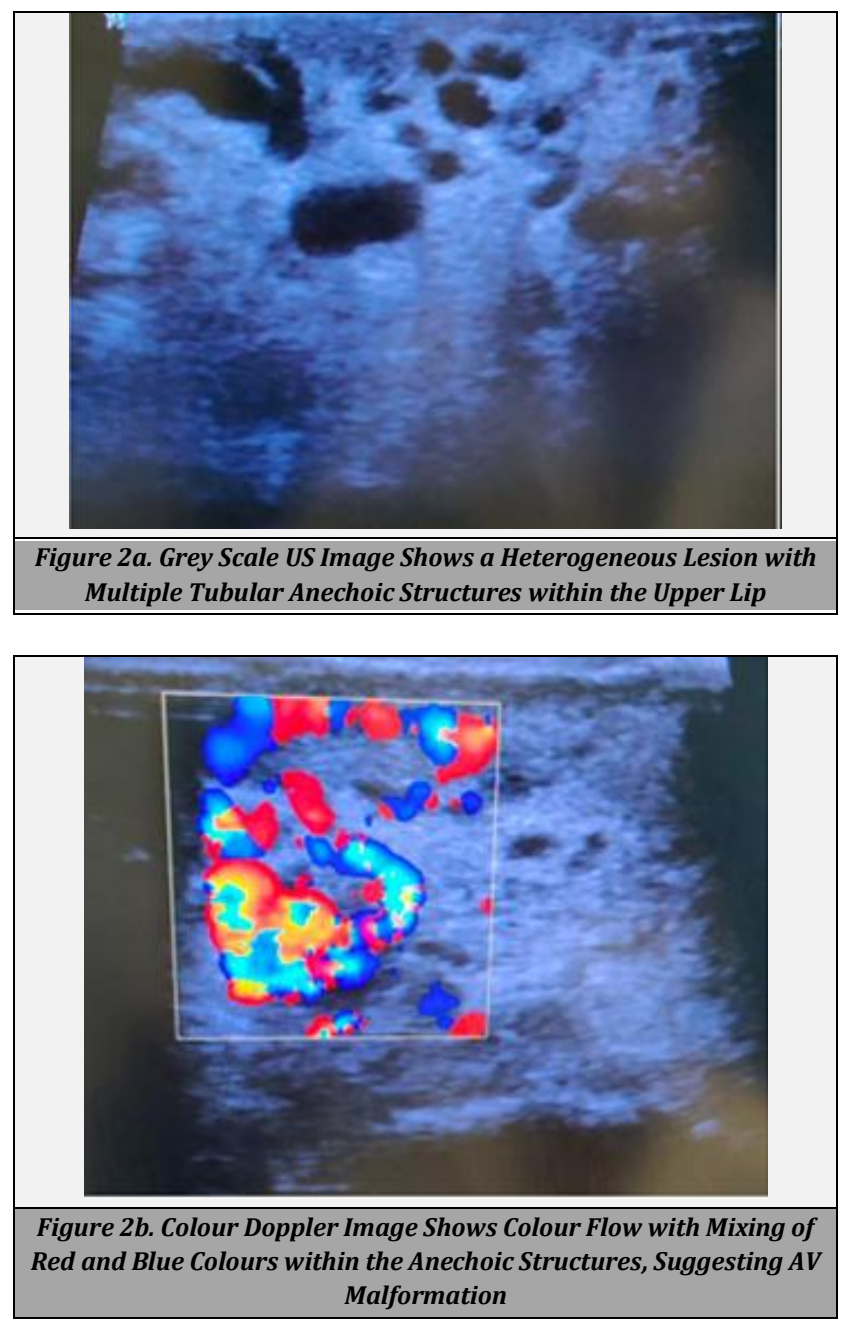

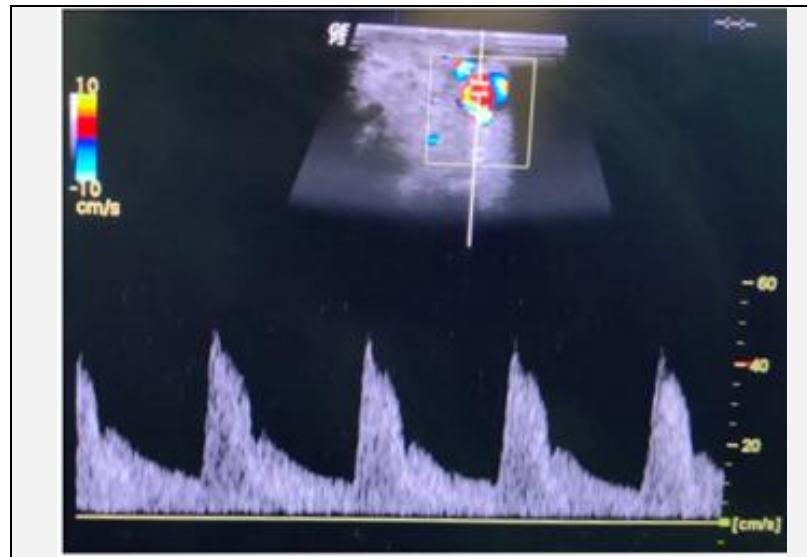

Figure 3. Colour Doppler Imaging with Spectral Waveform Shows High Velocity Flow with Low Resistance within the Nidus

On advice the patient underwent CECT examination of the head and neck for further evaluation of the lesion which was a soft tissue density lesion with multiple torturous vessels suggestive of nidus with arterial feeders and draining veins in the upper lip (Figure 4). Enlarged vessels were also noted in the dorsum of nose. Arterial feeders appeared to arise from the right external carotid artery. There was no obvious involvement of underlying bone.

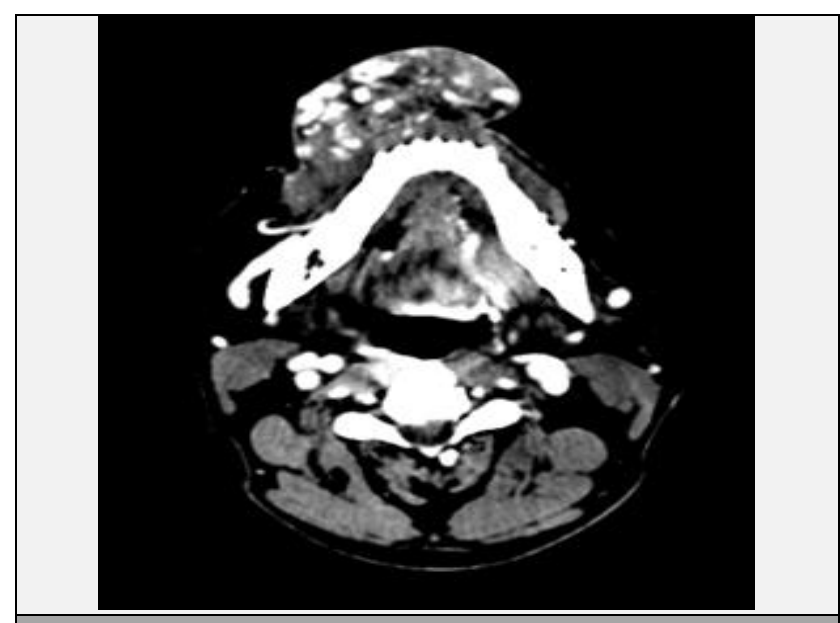

Figure 4. CECT of Head and Neck Shows a Soft Tissue Density Lesion with Multiple Torturous Vessels in the Upper Lip -Suggestive of Nidus

The patient was explained about the nature of the lesion and was advised angiographic studies for further evaluation. However, the patient was not willing for any further investigations.

\section{DISCUSSION}

Vascular lesions of head and neck have an estimated prevalence of $4.5 \%$ with most of these lesions being present since birth or childhood. ${ }^{5}$ Congenital vascular malformations occur as a result of abnormal vascular development whereas acquired vascular malformations can occur as a result of trauma or surgery. AV malformations are a group of vascular malformations characterised by a central nidus of abnormal arteriovenous channels and a normal intervening capillary bed. These are high flow vascular anomalies found most commonly in the spine and brain. The most common extra- 
cranial site includes the head and neck region, followed by limbs and trunk.

Clinically, they have a wide variety of presentations ranging from a simple port wine stain in involutional stage to a very prominent swelling with skin warmth and pulsations. Approximately $60 \%$ of these lesions are present at birth but they become apparent by 1 st or 2 nd decade of life. They do not have any sex predilection and may continue to grow throughout the lifetime. Most of these lesions are sporadic but they may also be found in association with syndromes like Cobb, Parkes-Weber and capillary malformations. ${ }^{6}$ Schobinger staging system divides the natural course of AV malformations into 4 different stages: 1) Quiescent phase, 2) Expansive phase, 3) Destruction phase and 4) Decompensate phase. ${ }^{7}$ Their natural progression is influenced by factors like hormonal changes, pregnancy, trauma etc. Histologically, these lesions are characterised by encapsulated aggregation of malformed feeder arteries and arterialisation of veins, which bypass the capillaries and form a nidus. There may be 1 or more feeder arteries or draining veins with arterial or venous networks. They are often associated with reactive angioproliferative components which explain their relapse after surgical removal. ${ }^{8}$ Long standing AV malformations are associated with local complications like increased risk of bleeding, thrombosis, skin ulceration, bone remodelling, deformity, compression of adjacent structures as well as systemic complications like cardiac failure.

Ultrasound and Colour Doppler Imaging (CDI) constitute the primary imaging for vascular lesions. The lack of ionising radiation, easy accessibility as well as their non-invasive nature makes ultrasonography an excellent initial modality of investigation. Colour Doppler Imaging (CDI) can be used to confirm the vascular nature of the lesion. Spectral waveform can also be used to demonstrate the high velocity flow with low resistance nature of the lesion. On ultrasound, AVM appears as heterogeneous lesion with multiple anechoic tubular structures which constitute the feeding and draining vessels. The arterial component of the lesion typically demonstrates a high systolic velocity with low resistance. Arterialised veins also demonstrate a pulsatile flow on spectral waveform which suggests the formation of $\mathrm{AV}$ communications.

Computed Tomography (CT) can be used to identify the vascular components of the lesion and also access the extent of the lesion. It can also be used to differentiate AV malformations from other vascular lesions. ${ }^{9}$ However CT has the disadvantage of ionising radiation because of which it has to be used only to assess complications related to AV malformations like acute bleeding, thrombosis, infections etc.

Magnetic Resonance Imaging (MRI) is a non-invasive modality of investigation which does not produce any ionising radiation. Because of lack of ionising radiation, it is safer to be used in paediatric population. It provides an excellent tissue contrast and can be used to assess the morphology as well as the pathophysiology of the lesions. On MRI, AV malformations are seen as multiple torturous dilated veins and hypertrophied arteries with low signal intensity and a characteristic lack of soft tissue component on $\mathrm{T} 1$ and $\mathrm{T} 2$ weighted images. The low signal intensity is attributed to the high flow velocity within these vessels. Gadolinium enhanced scans can also be used to delineate the vascular nature of the lesion and identify the feeder arteries and draining veins. Other MR imaging techniques like Time-of-flight (TOF) and Phase contrast can also be used to identify the vessels.

Another imaging technique is angiography which can used when the findings are in-conclusive in MRI or CT based studies. It provides information regarding the extent of the lesion, the feeder arteries and draining veins and also can be used as a guide for interventional procedures. On angiography, they typically appear as hypertrophied arteries which rapidly drain into draining veins through a nidus.

Management of AV malformations is challenging because of their increased tendency to recur. There has been considerable debate regarding the origin of recurrent lesions, i.e., if they are true recurrences or missed remnants. ${ }^{10}$ Surgical removal remained the mainstay of treatment for decades. With the advent of interventional radiology, techniques like endovascular embolization emerged as a better treatment option because of lower rates of complications especially in surgically difficult areas. Endovascular embolization with absolute ethanol has opened a new era of endovascular management of these lesions.11 Other procedures include sclerotherapy and laser coagulation. A multimodality approach including both surgical technique as well as interventional techniques remain the best treatment approach for these lesions.

The differential diagnosis of AV malformation includes other vascular malformations and vascular tumours like haemangiomas. Unlike AV malformations, vascular tumours show soft tissue enhancement.

\section{CONCLUSIONS}

AV malformations of head and neck are group of vascular lesions which can affect a person both psychologically as well as physically. A clear understanding of the pathophysiology and an appropriate radiological diagnosis can greatly influence the management of these lesions and their outcome. Prompt identification of these lesions is also required to avoid unnecessary complications and thereby improve its prognosis.

Financial or other competing interests: None.

Disclosure forms provided by the authors are available with the full text of this article at jemds.com.

\section{REFERENCES}

[1] Cohen MM Jr. Vascular update: morphogenesis, tumors, malformations and molecular dimensions. Am J Med Genet A 2006;140(19):2013-38.

[2] Boon LM, Ballieux F, Vikkula M. Pathogenesis of vascular anomalies. Clin Plast Surg 2011;38(1):7-19.

[3] Dasgupta R, Fishman SJ. ISSVA Classification. In: Seminars in pediatric surgery. WB Saunders 2014;23(4):158-61.

[4] Mulliken JB, Fishman SJ, Burrows PE. Vascular anomalies. Curr Probl Surg 2000;37(8):517-84.

[5] Lee JW, Chung HY. Vascular anomalies of the head and neck: current overview. Arch Craniofac Surg 2018;19(4):243-7.

[6] Miller DD, Gupta A. Histopathology of vascular anomalies: update based on the revised 2014 ISSVA classification. 2016;35(3):137-46. 
[7] Liu AS, Mulliken JB, Zurakowski D, et al. Extracranial arteriovenous malformations: natural progression and recurrence after treatment. Plast Reconstr Surg 2010;125(4):1185-94.

[8] Ginanni B, Lauretti DL, Vallini V, et al. Arterio-Venous Malformations (AVMs): imaging findings in a wide spectrum of localizations. European Congress of Radiology-ECR 2013.

[9] Pandhare MN, Jyoti DB, Mandale MS, et al. Acquired arteriovenous malformation of lip occurring as an occupational hazard: a case report with review of literature. J Oral Maxillofac Pathol 2018;22(2):287.
[10] Aboukaïs R, Vinchon M, Quidet M, et al. Reappearance of arteriovenous malformations after complete resection of ruptured arteriovenous malformations: true recurrence or false-negative early postoperative imaging result? J Neurosurg 2017;126(4):1088-93.

[11] Yakes WFJ. Endovascular management of high-flow arteriovenous malformations. Vol. 21. In: Seminars in Interventional Radiology. Thieme Medical Publishers 2004: p. 49. 